\title{
Impact of nasal ventilation on survival in hypercapnic Duchenne muscular dystrophy
}

\author{
A K Simonds, F Muntoni, S Heather, S Fielding
}

\begin{abstract}
Background-Respiratory failure is the commonest cause of death in patients with Duchenne muscular dystrophy (DMD). Life expectancy is less than one year once diurnal hypercapnia develops. This study examines the effects of nasal intermittent positive pressure ventilation (NIPPV) on survival in symptomatic Duchenne patients with established ventilatory failure. Methods-Nocturnal NIPPV was applied in 23 consecutive patients with DMD of mean (SD) age 20.3 (3.4) years who presented with diurnal and nocturnal hypercapnia.
\end{abstract}

Results-One year and five year survival rates were $85 \%(95 \%$ CI 69 to 100$)$ and $73 \%$ (95\% CI 53 to 94), respectively. Early changes in arterial blood gas tensions following NIPPV occurred with mean (SD) $\mathrm{Po}_{2}$ increasing from $7.6(2.1) \mathrm{kPa}$ to 10.8 (1.3) $\mathrm{kPa}$ and mean (SD) $\mathbf{P C O}_{2}$ falling from $10.3(4.5) \mathrm{kPa}$ to $6.1(1.0) \mathrm{kPa}$. Improvements in arterial blood gas tensions were maintained over five years. Health perception and social aspects of SF-36 health related quality of life index were reported as equivalent to other groups with nonprogressive disorders using NIPPV.

Conclusion-Nasal ventilation is likely to increase survival in hypercapnic patients with Duchenne muscular dystrophy and should be considered as a treatment option when ventilatory failure develops.

(Thorax 1998;53:949-952)

Sleep and Ventilation Unit, Respiratory Support Service, Royal Brompton and Harefield NHS Trust, London SW3 6NP, UK

A K Simonds

$S$ Heather

$S$ Fielding

Department of

Paediatrics and

Neonatal Medicine,

Hammersmith

Hospital, London W12,

UK

F Muntoni

Correspondence to:

Dr A K Simonds.

Received 9 February 1998

Returned to author

1 May 1998

Revised manuscript received

8 June 1998

Accepted for publication

17 June 1998
Keywords: Duchenne muscular dystrophy; nasal intermittent positive pressure ventilation; mortality

In patients with Duchenne muscular dystrophy (DMD) a characteristic pattern of respiratory function is seen during childhood and adolescence. Vital capacity (VC) increases as predicted until around 10 years of age. A plateau then occurs followed by a steady fall in $\mathrm{VC}$ as respiratory muscle weakness progresses, accompanied by the development of a thoracic scoliosis in the majority of cases. The annual decrease in VC may be as much as $250 \mathrm{ml}$ in the late teenage years. ${ }^{1}$ Once VC falls below $20 \%$ predicted ventilatory failure is almost inevitable, with $73 \%$ of DMD patients dying of respiratory insufficiency. ${ }^{2}$ About $10 \%$ die of a cardiomyopathy which may be exacerbated by chronic hypoxaemia and hypercapnia. Hypoventilation, sometimes associated with obstructive apnoeas, ${ }^{3}$ first occurs during sleep as a result of the reduction in intercostal and accessory muscle tone, and a decrease in ven- tilatory drive which is most prominent in rapid eye movement (REM) sleep. Untreated, nocturnal hypoventilation progresses to daytime ventilatory failure and ultimately cor pulmonale. Acute hypercapnic exacerbations complicate the clinical course and are usually caused by chest infections as inspiratory muscle weakness predisposes the individual to atelectasis, and cough is impaired by expiratory muscle insufficiency.

A study in which normocapnic DMD patients with a vital capacity of $20-50 \%$ predicted were randomised to receive nasal ventilation or a control group without ventilatory support showed that nasal ventilation had no beneficial effect on survival and was poorly tolerated. ${ }^{4}$ By contrast, mean survival after diurnal hypercapnia develops in DMD is 9.7 months without ventilatory assistance. ${ }^{5}$ In patients with established ventilatory failure nasal ventilation can be life saving. ${ }^{5}$ It has been argued that it is unethical to deny ventilatory assistance to hypercapnic DMD patients as death is the inevitable consequence of withholding treatment. ${ }^{6}$ Information on the survival and quality of life in patients with DMD with hypercapnic respiratory failure receiving assisted ventilation is sparse. There are reports of negative pressure ventilation in $\mathrm{DMD},{ }^{7-11}$ although this is not the treatment of choice as upper airway obstruction during sleep may be provoked. ${ }^{11}$ Mouth ventilation has been successful in some centres ${ }^{12}$ but long term survival using non-invasive techniques is not well documented. In the largest study of NIPPV in DMD 16 patients were treated initially with nasal ventilation, but five were transferred to tracheostomy ventilation (T-IPPV).$^{13}$ Despite the fact that nasal ventilation was pioneered in patients with $\mathrm{DMD},{ }^{14}$ a progressive care plan of non-invasive ventilation followed by T-IPPV, or T-IPPV alone, is the norm in many European countries. This complicates management as tracheostomy ventilation is more difficult to deliver in the home than noninvasive ventilation and may delay or prevent discharge. In addition, most patients and their carers prefer non-invasive methods where these can be used safely. ${ }^{15}$ In this study hypercapnic DMD patients have been treated solely with long term domiciliary nasal ventilation to examine the impact of NIPPV on survival, arterial blood gas tensions, and health related quality of life.

\section{Methods}

Twenty three consecutive patients referred to the Royal Brompton Hospital are included in the analysis. A diagnosis of DMD was made 
Table 1 Patient characteristics on starting nasal ventilation $(n=23)$

\begin{tabular}{lcll}
\hline & Values & \multicolumn{2}{c}{ Range } \\
\hline Age (years) [median] & 20.3 & $(3.4)[20.0]$ & $13-28$ \\
$\mathrm{PaO}_{2}(\mathrm{kPa})$ & 7.57 & $(2.1)$ & $4.0-12.8^{\star}$ \\
$\mathrm{PaCO}_{2}(\mathrm{kPa})$ & 10.26 & $(4.5)$ & $6.4-24$ \\
Vital capacity (ml) & 306 & $(146)$ & $0-600$ \\
\hline
\end{tabular}

Values are mean (SD)

* Measured on supplemental oxygen therapy.

according to standard criteria. ${ }^{16}$ All patients were non-ambulant by the age of 12 years and none had features of Becker muscular dystrophy. Five were referred from intensive care units elsewhere with uncontrolled acute on chronic hypercapnic respiratory failure. After weaning using nasal ventilation, domiciliary ventilation was initiated if diurnal hypercapnia and severe symptomatic nocturnal hypoventilation $\left(\mathrm{PCO}_{2}>7 \mathrm{kPa}\right)$ persisted in a steady state period on recovery. Eighteen patients were treated electively for symptomatic daytime hypercapnia and confirmed nocturnal hypo-

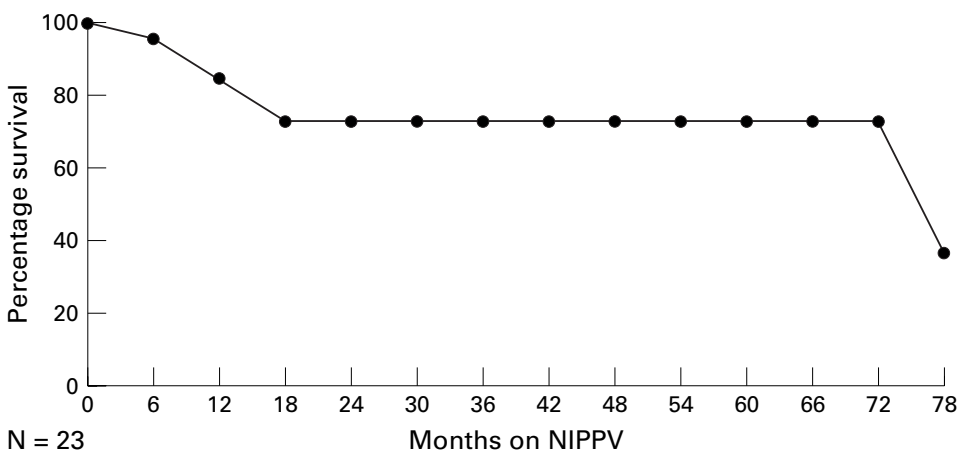

Figure 1 Survival in hypercapnic patients with Duchenne muscular dystrophy using nasal intermittent positive pressure ventilation.
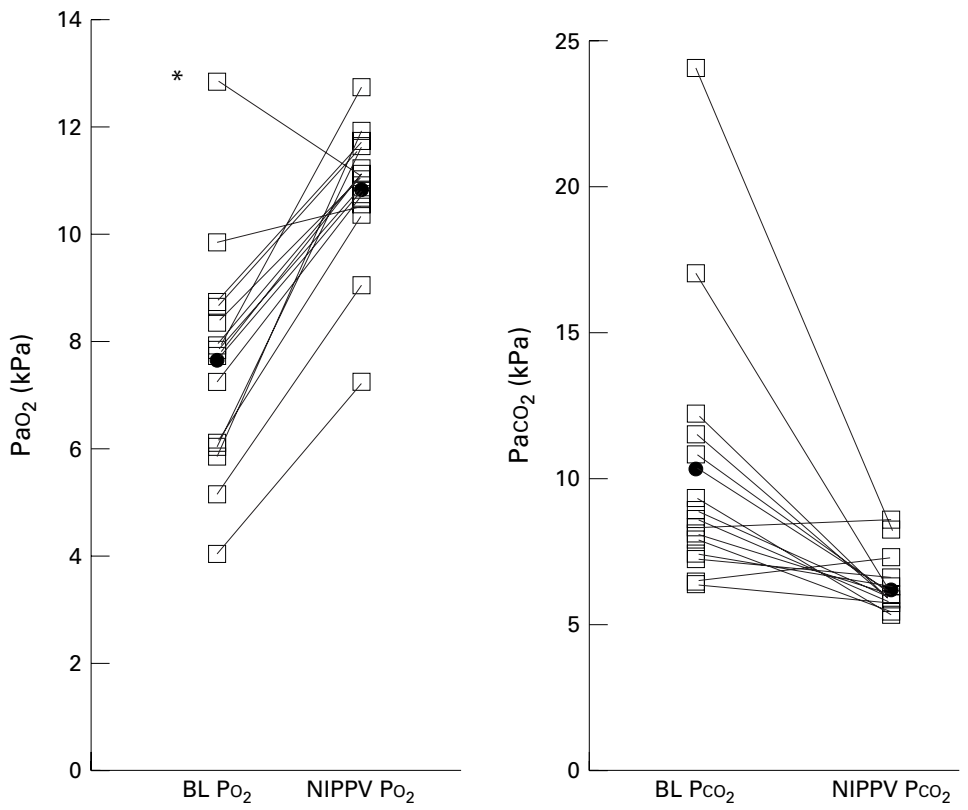

Figure 2 Effect of nasal intermittent positive pressure ventilation on arterial blood gas tensions in all patients. $\mathrm{BLP \textrm {O } _ { 2 }}=$ baseline arterial oxygen tension; $\mathrm{BLCO}_{2}=$ baseline arterial carbon dioxide tension; $N I P P V P_{2}=$ arterial oxygen tension breathing spontaneously after starting NIPPV; NIPPVPCO $\mathrm{CO}_{2}=$ arterial carbon dioxide tension breathing spontaneously after starting NIPPV. ventilation. Vital capacity was unrecordable at the start of nasal ventilation in 10 patients.

The patients all used domiciliary nasal ventilators at night in assist/control mode. No preference was given to any particular type of nasal ventilator as a previous study in this unit using pressure preset and volume preset devices produced similar effects on arterial blood gas control and respiratory muscle effort. ${ }^{17}$ Twenty subjects used pressure preset devices, 16 Nippy (Friday Medical, London, UK), two BiPAP (Respironics Inc, Murrysville, USA), two DP90 (Taema, Deva Medical, Runcorn, UK) and three used volume preset models (PLV-100, Lifecare, Lafayette Co, USA or BromptonPAC, Pneupac Ltd, Luton, UK). Commercial nasal interfaces, the Rescare bubble mask, (Resmed Ltd, Abingdon, UK), Respironics nasal mask, (Respironics, Murrysville, USA) or Adams circuit, (Puritan Bennett, Hounslow, UK ) were employed according to patient preference. Ventilation was started in hospital in all cases and overnight monitoring of arterial oxygen saturation $\left(\mathrm{SaO}_{2}\right)$ and transcutaneous $\mathrm{CO}_{2}$ was carried out to optimise control of nocturnal hypoventilation with the aim of maintaining $\mathrm{SaO}_{2}$ at $>90 \%$ and transcutaneous $\mathrm{CO}_{2}$ in the normal range. ${ }^{18}$ Inspiratory pressures of $16-28 \mathrm{~cm} \mathrm{H}_{2} \mathrm{O}$ were used and expiratory positive pressures of $2-5 \mathrm{~cm} \mathrm{H}_{2} \mathrm{O}$ where the latter was available.

Patients were advised to use domiciliary nasal ventilation during sleep at night. Supplemental oxygen therapy was not used. Carers and family members were taught to perform daily chest physiotherapy for the patients during nasal ventilation to improve sputum clearance. A modified active cycle of breathing technique was used to achieve effective coughing. Inspiratory pressure or tidal volume were temporarily increased by around $20 \%$ during physiotherapy sessions to increase inspiratory volume, and manual chest clapping and shaking were combined with assisted coughing/huffing. ${ }^{19}$ Humidification via a heated water bath system (Resmed) was added in some patients at the time of acute chest infections, but was not used long term. Outpatient review was carried out every 3-6 months.

Ventilators were serviced in the patient's home at 6-12 month intervals and all patients/ carers had access to a hospital 24 hour telephone respiratory support hotline service to report equipment problems or seek medical advice. Thirteen of 18 surviving patients completed the SF-36 questionnaires on health related quality of life 3-72 months after starting nasal ventilation.

\section{Results}

Patient characteristics before the start of nasal ventilation are given in table 1 . All patients tolerated nasal ventilation and none requested to discontinue therapy. Kaplan-Meier analysis showed one year survival was $85 \%$ (95\% CI 69 to 100 ) with $73 \%$ survival ( $95 \%$ CI 53 to 94 ) at two years which was maintained at five years (fig 1). Arterial $\mathrm{PO}_{2}$ and $\mathrm{PCO}_{2}$ by the time of discharge improved significantly on NIPPV (fig 2) and these improvements were sustained over 


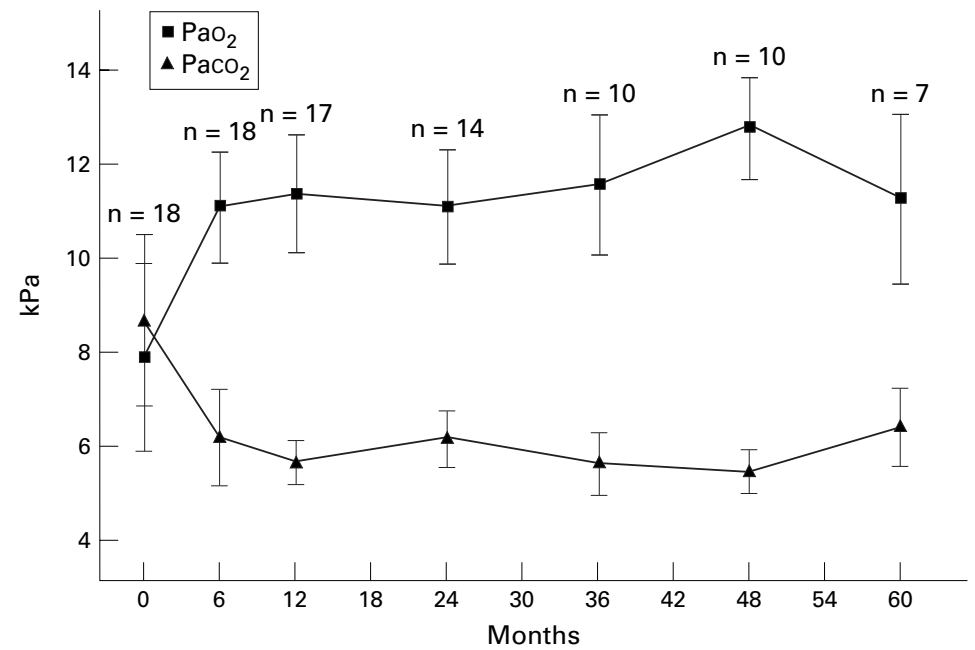

Figure 3 Evolution of arterial blood gas tensions during treatment with NIPPV in patients treated electively (diurnal values obtained with patients breathing air spontaneously).

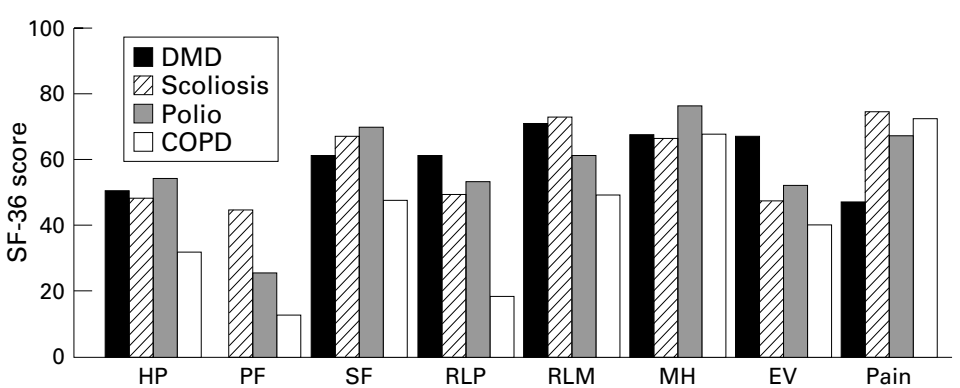

Figure 4 Health related quality of life SF-36 results. DMD $=$ Duchenne muscular dystrophy; Polio $=$ post polio patients; $C O P D=$ chronic obstructive pulmonary disease (all on NIPPV);HP = health perception; $P F=$ physical function; $S F=$ social function; $R L P=$ role limitation related to physical factors; RLM = role limitation related to mental factors; $M H=$ mental health $E V=$ energy and vitality. $0=$ minimum score $; 100=$ maximum score except for pain where $0=$ maximum pain and $100=$ no pain.

time (fig 3). Median duration of NIPPV use was 9.5 hours per day. Patients continued with nocturnal NIPPV for 5-7 years. Increasing ventilatory dependency then developed in three subjects but no patient developed bulbar weakness which precluded normal feeding. One patient had a percutaneous gastrostomy sited to augment oral nutrition.

SF-36 quality of life results are given in fig 4 compared with groups with idiopathic scoliosis, chronic obstructive pulmonary disease (COPD), or post polio ventilatory failure receiving NIPPV at the Royal Brompton Hospital. For the patients with DMD physical function was severely reduced, but domains such as mental health, role limitation related to physical and emotional factors, and social function did not differ significantly from age matched male controls. ${ }^{20}$ Health perception was superior to that of older patients on NIPPV with a progressive condition such as COPD.

After starting NIPPV the average hospital admission rate for each patient was 0.64 per year with a mean (SD) hospital stay of 4.0 (4.3) days. Most admissions were for treatment of chest infections. No patient required endotracheal intubation or a tracheostomy during an acute episode.
Seven patients undertook university degree or higher education courses during treatment and three continued at mainstream school. Three patients developed left ventricular failure due to dilated cardiomyopathy which was treated with diuretic and ACE inhibitor therapy. Deaths were due to respiratory failure in five cases at a mean of 23.8 months after starting NIPPV.

\section{Discussion}

Domiciliary nasal ventilation is established treatment for patients with ventilatory failure due to restrictive chest wall disease and non-progressive neuromuscular conditions such as previous poliomyelitis affecting respiratory muscles. ${ }^{13}{ }^{21}$ Previously it was thought that non-invasive ventilation could not be used as the sole means of ventilatory support in patients with DMD as loss of bulbar function and poor expiratory muscle strength would necessitate tracheostomy ventilation later in the course of the disease. ${ }^{813}$ Ethical concerns have been expressed that the use of NIPPV in a progressive disease such as DMD would be a burden to the patient and family, and likely to result in poor survival and a severely impaired quality of life. The findings presented here contradict these widely held views.

Although an uncontrolled series, the results confirm that long term survival can be achieved with NIPPV. This impact of NIPPV on prolongation of life can only be speculative as we felt it unethical to include an unventilated group of hypercapnic DMD patients. However, previous studies ${ }^{25}$ have consistently shown a life expectancy of around 20 years in untreated patients, suggesting that NIPPV may extend survival in some patients by five years or more. Direct comparison with the outcome in patients with DMD who have received T-IPPV is difficult as many are treated with a range of ventilatory methods before a tracheostomy is carried out.

Quality of life in NIPPV recipients is comparable to other patient groups with non-progressive disorders, despite a lower level of physical function. The effect of NIPPV on the quality of life of the patient's family/carers remains to be assessed. Hospitalisation rates were generally low and no patient required admission to the intensive care unit.

NIPPV offers the advantage of allowing normal speech and feeding, and avoids complications related to a tracheal stoma such as tracheal stenosis and nosocomial infection. Volume preset ventilators have been used by previous workers ${ }^{13}$ but this study would suggest that pressure preset ventilators are at least as effective. Some patients may benefit from a combination of mouth ventilation and NIPPV. Meticulous attention to physiotherapy during NIPPV is essential in patients with profoundly weak expiratory muscles, and is crucial to the success of the technique. Bach et $a l^{2}$ have shown that the combination of non-invasive ventilation and cough aids produces lower hospitalisation rates than T-IPPV, although survival rates are not given. T-IPPV may still be needed in some individuals, but progression 
from non-invasive to invasive ventilation should not be seen as inevitable. This is important as T-IPPV is technically more demanding to deliver in the home than nasal ventilation, and it is much more difficult for individuals to attend higher education courses or to travel if they are dependent on T-IPPV. It should also be acknowledged that in some countries there is a reluctance to use tracheostomy ventilation in progressive neuromuscular disease, although in this study T-IPPV remained an option if problems developed with nasal ventilation.

Negative pressure ventilation $(\mathrm{nPV})$ is a non-invasive alternative to NIPPV. ${ }^{7-10}$ Using body suit $\mathrm{nPV}$ a maximum survival of four years was seen in one series of nine patients with $\mathrm{DMD},{ }^{10}$ but the increase in diurnal $\mathrm{PaO}_{2}$ did not match that obtained in the present study. Overnight monitoring during nPV has confirmed recurrent episodes of arterial oxygen desaturation due to obstructive apnoeas which were abolished by the addition of nasal continuous positive airway pressure, but not by supplemental oxygen therapy. ${ }^{11}$ By promoting upper airway patency during sleep, NIPPV is likely to control sleep disordered breathing more effectively than nPV resulting in improved diurnal arterial blood gas tensions. This mechanism could explain the survival advantage seen with NIPPV.

The results suggest that the option of nasal ventilation should be available to all patients with DMD who choose to receive ventilatory support. The study can be criticised in that measurements of health status and respiratory muscle strength before and after NIPPV would have been preferable; however, many patients were too ill to complete these assessments on admission. It will only be possible to obtain serial assessment before and after NIPPV if patients are referred for respiratory management before the development of ventilatory failure.

An interesting feature is the plateau in survival over years one to four. More work is required to examine whether the improvement in arterial blood gas tensions leads to a stabilisation in respiratory muscle strength and/or cardiac function over this period.

Finally, a significant number of patients presented in uncontrolled ventilatory failure, having been lost to medical follow up since discharge from paediatric care. Most patients had experienced symptoms of nocturnal hypoventilation (headaches, poor sleep quality, anorexia, breathlessness) and an increased frequency of chest infections for a few years before developing ventilatory failure. Many teenage patients had not transferred adequately from paediatric to adult medical care services, or adult respiratory follow up had been discontinued. Improved liaison between paediatri- cians and adult physicians is likely to reduce the number of patients with DMD (and those with other congenital muscular dystrophies and myopathies) who develop uncontrolled ventilatory failure, and to allow patients and their families to consider ventilatory options in advance. This process may be facilitated by the establishment of adolescent neuromuscular clinics where neurological, respiratory, cardiac and orthopaedic care, plus nutritional and physiotherapy advice can be co-ordinated.

1 Baydur A, Gilgoff I, Prentice W, et al. Decline in respiratory function and experience with long term assisted ventilation in advanced Duchenne's muscular dystrophy. Chest 1990;97:884-9

2 Rideau Y, Gatin G, Bach J, et al. Prolongation of life in Duchenne's muscular dystrophy. Acta Neurol 1983;5:11824.

3 Khan Y, Heckmatt JZ. Obstructive apnoeas in Duchenne muscular dystrophy. Thorax 1994;49:157-61.

4 Raphael J-C, Chevret S, Chastang C, et al. Randomised trial Raphael J-C, Chevret S, Chastang C, et al. Randomised trial
of preventive nasal ventilation in Duchenne muscular dysof preventive nasal ventilation in
trophy. Lancet 1994;343:1600-4.

5 Vianello A, Bevilacqua M, Salvador V, et al. Long-term nasal intermittent positive pressure ventilation in advanced Duchenne's muscular dystrophy. Chest 1994;105:445-8.

6 Hill NS. Noninvasive positive pressure ventilation in neuromuscular disease. Enough is enough. Chest 1994;105:3378.

7 Curran FJ. Night ventilation by body ventilators for patients in chronic respiratory failure due to late stage Duchenne muscular dystrophy. Arch Phys Med Rehabil 1981;62:2704.

8 Alexander MA, Johnson EW, Petty J, et al. Mechanical ventilation of patients with late stage Duchenne muscular
dystrophy: management in the home. Arch Phys Med Rehabil 1979;60:289-92.

9 Splaingard ML, Jefferson LS, Harrison GM. Survival of patients with respiratory insufficiency secondary to neuromuscular disease treated at home with negative pressure ventilation (abstract). Am Rev Respir Dis 1982;125:139.

$10 \mathrm{Mohr}$ CH, Hill NS. Long term follow-up of nocturnal ventilatory assistance in patients with respiratory failure due to Duchenne-type muscular dystrophy. Chest 1990;97:91-6.

11 Hill NS, Redline S, Carskadon M, et al. Sleep-disordered breathing in patients with Duchenne muscular dystrophy using negative pressure ventilators. Chest 1992;102:16562.

12 Bach JR, Alba AS, Saporito LR. Intermittent positive pressure ventilation via the mouth as an alternative to tracheostomy for 257 ventilator users. Chest 1993;103:174-82.

13 Leger P, Bedicam JM, Cornette A, et al. Nasal intermittent positive pressure ventilation. Long term follow-up in patients with severe chronic respiratory insufficiency. Chest 1994;105:100-5.

14 Rideau Y, Delaubier A. Management of respiratory neuromuscular weakness. Muscle Nerve 1988;11:407-8.

15 Bach J. A comparison of long-term ventilatory support alternatives from the perspective of the patient and alternatives from the perspective

16 Baker E, Jennekens FGI, de Visser M, et al. Duchenne and Becker muscular dystrophies. In: Emery AEH, ed. Diagnostic criteria for neuromuscular disorders. London: Royal Society of Medicine Press, 1997: 1-4

17 Elliott MW, Aquilina R, Green M, et al. A comparison of different modes of ventilatory support : effects on ventilation and inspiratory muscle effort. Anesthesia 1994; 49:279-83.

18 Simonds AK. Paediatric non-invasive ventilation. In: Simonds AK, ed. Non-invasive respiratory support. London: Chapman \& Hall Medical, 1996: 102-15.

19 Bott J, Moran F. Physiotherapy and nasal intermittent positive pressure ventilation. In: Simonds AK, ed. Non-invasive respiratory support. London: Chapman \& Hall Medical, respiratory suppor

20 Jenkinson C, Wright L, Coulter A. Quality of life measurement in health care. A review of measures and population norms for the UK SF-36. Oxford: Health Services Research Unit, 1993: 32-42.

21 Simonds AK, Elliott MW. Outcome of domiciliary nasal intermittent positive pressure ventilation in restrictive and obstructive disorders. Thorax 1995;50:604-9.

22 Bach JR, Ishikawa Y, Kim H. Prevention of pulmonary morbidity for patients with Duchenne muscular dystrophy. Chest 1997;112:1024-8. 